\title{
Violence Detection and Target Finding using Computer Vision
}

\author{
A. Sangeerani Devi, Sonia Prakash, K. Laavanya, A.Shali, D.Sathish Kumar
}

\begin{abstract}
Recognizing savagery in recordings through CCTV is basic for requirement and investigation of reconnaissance cameras with the plan of keeping up open wellbeing. Moreover, it will be an incredible device for securing kids and help guardians settles on a superior educated choice about their children. However, this can be a difficult drawback since detecting certain nuances with no human administration isn't entirely technical however also a conceptual problem.So, our idea is to use computer vision to develop an automated technique in detecting the violent behavior/street crime criminals through surveillance cameras installed in cities and towns. When the surveillance cameras detect the abnormal behavior, it captures the scene and generates an alert by sending the captured image to the nearby police station. Further, the CCTV cameras using Cloud trigger the near-by cameras to track the particular target and its location.
\end{abstract}

Keywords: ViolenceDetection, Surveillance Cameras, Capture, Trigger,Google Cloud.

\section{INTRODUCTION}

Violence is a global public health issue and in public spots vigorousbehaviors lead to a severe threat toindividual security and social soundness. It continuously demands efforts from authorities to provide the populace with safer public location at present, a large of equipment are applied in public places, prompting enormous measure concerning on the protection orderlies. Therefore, it is of high importance to automatically identify viciousnessevents from CCTV.Additionally, ongoing years, we've seen that there has been a stamped and continued development in the utilization of CCTV observation cameras so as to forestall wrongdoings in open spots. In the midst of the related open consumption and the development, just as worries about their social expenses and adequacy, there is an expanding requirements for a proof-based way to deal with educate CCTV practices and policies. Instances of badgering in working environments are additionally ending up intense. With the regularly developing establishment of cutting-edge CCTV framework, practically whole urban communities

Revised Version Manuscript Received on Jun 20, 2019.

A SANGEERANI DEVI, Computer science and Engineering, SriSairam Engineering College, Chennai, India.

SONIA PRAKASH, Computer science and Engineering, SriSairam Engineering College, Chennai, India.

K. LAAVANYA, Computer science and Engineering, SriSairam Engineering College, Chennai, India.

A.SHALI, Computer science and Engineering, SriSairam Engineering College, Chennai, India.

D.SATHISH KUMAR, Computer science and Engineering, SriSairam Engineering College, Chennai, India. would now be able to be observed, through the real reason served by the equivalent is simply evidential. It would just be normal to anticipate an alarm or cautioning framework for progressing (or going to occur) accidents and violations, where auspicious activity can be the distinction between life and demise. Such situations are probably going to be checked and recognized by workforce seeing live footage. Be that as it may, as the quantity of CCTV's per unit continues rising, this methodology is winding up progressively unreasonable. Consequently, what we require is a reconnaissance unit fit for flourishing in these circumstances with negligible human information.

The surveillance camera will be activated for recording just when there is a human presence. The camera is inactive when there is no individual existence. Human existence is checked utilizing a movement location calculation. When a crime is about to be commit, then the scene is captured by the CCTV and send to an adjacent police station. Consequently, creating an alarm system.

\section{PROPOSED SYSTEM}

The planned system cope with the present pattern of security frameworki.e. consolidation of protection concern, cost-viability, effective caution system. Restrictive video recording is important fuse.Consistently the camera is on but not recorded. It would continue setting up the edges it gets and wouldn't get it until it is "Activated".

The Activationis done only when many peopleare detected. Theseinvolve image processing with the blend to distinguish the essential instances. Once the gauge divergence is identified, then the scene is marked as vital thenit triggers the recording. It works as follows:

1) If the individual isn't the only one, we initiate the camera to blanked out/obscured any documentation, writing on a load up to keep away from any holes.

2) When it has been set up that the individual isn't the only one, we refine our investigation to recognizing the number of individuals, their sexual orientation and different attributes. We utilize human discovery, signal acknowledgment, and movement identification.

3) Voice is also not recorded. So, the tracking and human recognition are perpetually operative in a restrictive focus mode. Inrestrictive focus, we run only initial test for scene which is marked vital. When it is identified,we acquire heavier classifiers - Video and Audio processing. This ensures a smoother activity. 
The approach is to hang tight for a trigger to a fundamental circumstance and after that to make the required move. Thus, the likelihood of wrongdoing going unnoticedis fundamentally smothered even if false alerts are extended. The initial indicatorscan be quick and jerky movements betweenindividuals closer than specific safe closeness. Once recording is initiated in addition to an alert flag is issued either legitimate to the experts.

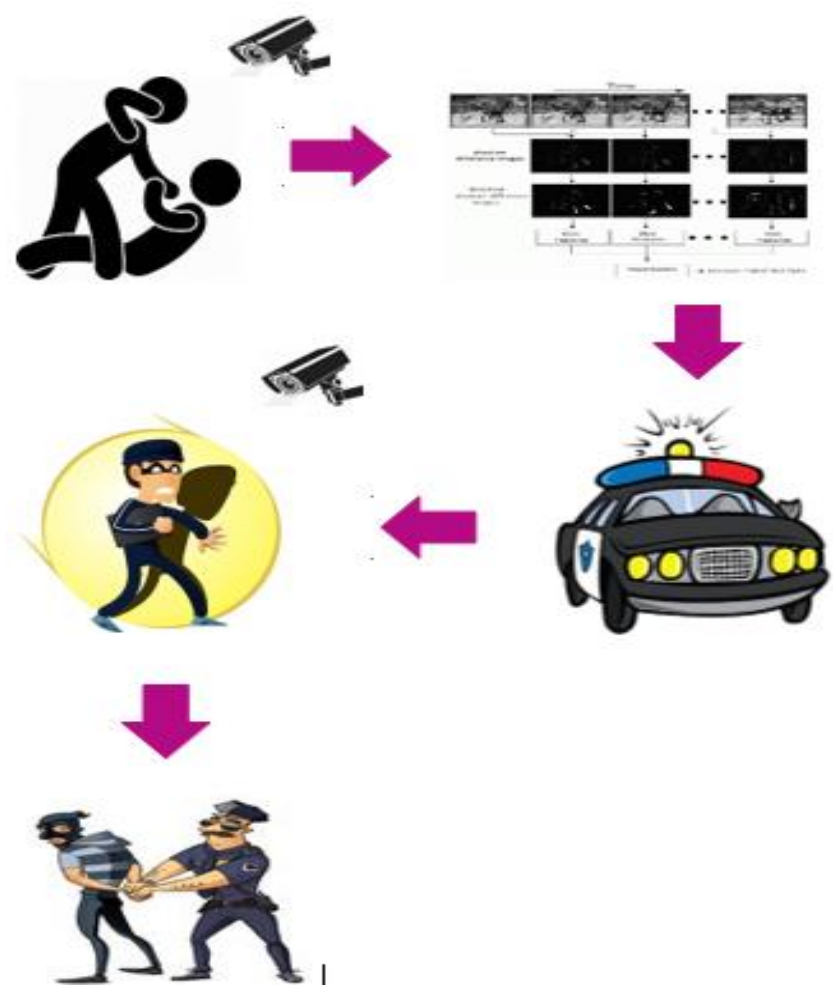

\section{CRIME DETECTION AND CAPTURING TARGET}

\section{METHODOLOGY}

Our goal is to structure and execute a framework to abstain from wrongdoing by pursuing human behavior through the investigation of video caught utilizing CCTV.

\section{A. Capturing Device}

The vitalscene is monitored by CCTV.The scene isn't recorded untilit is necessary i.e. video is recorded only when person's square measure unexpected movements or actions. Public configurations of CCTV systems had essential impac ts on crime.

\section{B. Capturing Of Frame}

Videos are combination of a sequence of images to form a moving picture. Frames are caught andhandled to picture for movement inside the scene. The movement in the scene indicate some act within the scene . A human presence is affirmedby appearances or shape designs.

\section{Detection}

Movement inside the scene is distinguished by examiningthe frames caught.This is accomplished by background subtraction where the still objects in the frame are removed by analysing the posture of every object. The object is identified as moving, if it's posture changes. Appearances or shape designsare matched with already saved activity, to confirm it is a human.

\section{Analysis}

In analysis, weprocess on standard tracking algorithm. We use the background subtraction which is based on Gaussian theory to section out the mobile instance. The movement monitoring is accomplished with the aid of Kalman filter. After inspecting the instance for all frames, the Filter then roughly calculate theinstance's pose in successive frames. At that point conduct of moving character is broke down utilizing social examination calculation to check for any demonstration of maltreatment, tormenting or badgering.

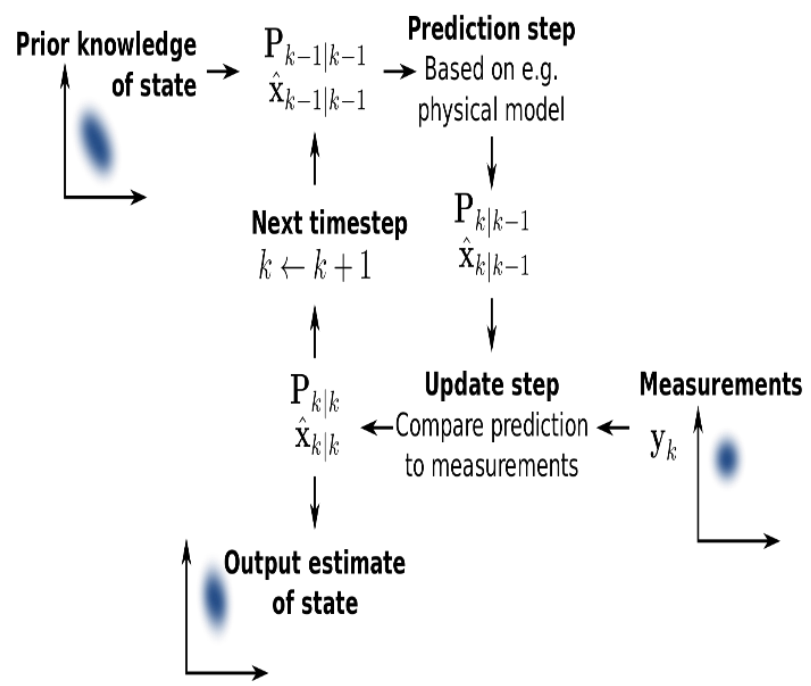

Kalman Filter

Kalman channel monitors the measurable condition of the framework and also the difference or uncertainty of the estimate. The estimate is updated by employing a state transition model and measurements. $X_{\mathrm{k} \mid \mathrm{k}-1}$ indicate the estimate of the system's state at step time $\mathrm{k}$ before the kth measurement $Y_{k}$ has been taken into consideration. $P_{k \mid k-1}$ is the corresponding uncertainty.

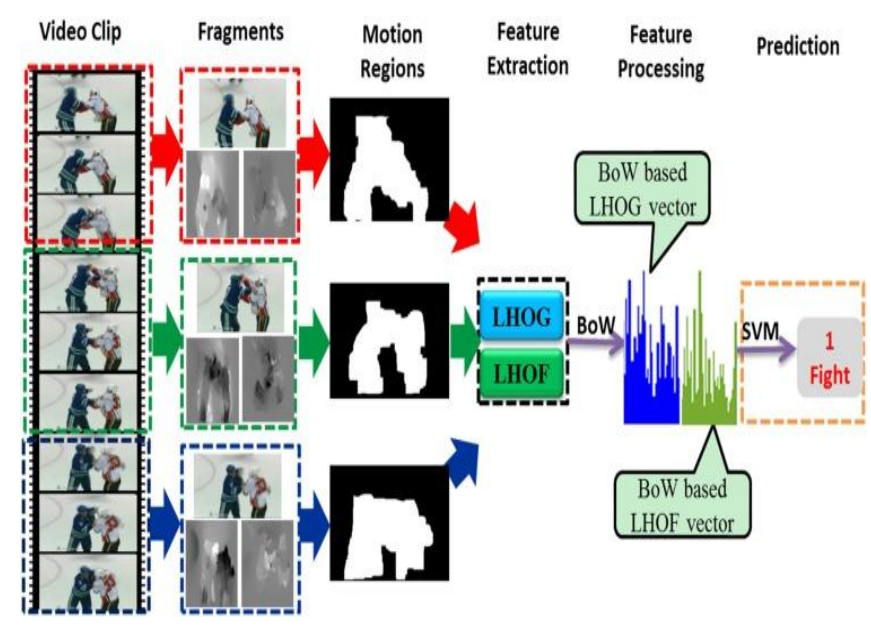

\section{PROCESSING OF VIDEO CLIP}

\section{E. Hmdb51}

In Human Motion Database, there are 6847 video cuts in this dataset, and these clips are isolated into certain activity classifications. These activity are categorized as follows:

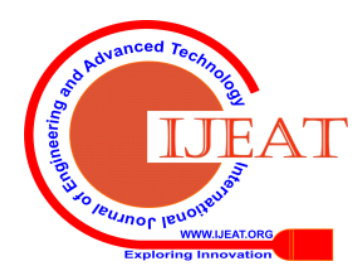


General facial activity

Shape motion and Shape motion with object interaction

Shape action for human interaction

A Multi-level reviewing of video is done to assess set of clips. Just those video tests are appraised "great" that have the standard that you can recognize the single fingers all through the movement. Those examples which don't meet this prerequisite are evaluated either "terrible" or "medium" if a portion of the appendages or body parts disappear while the activity is being executed.

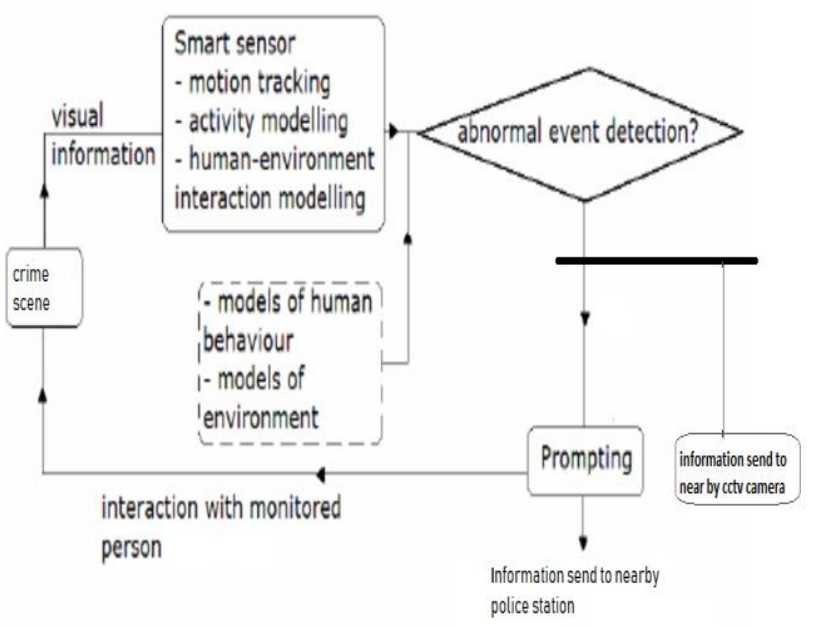

SYSTEM ARCHITECTURE

\section{DEVICE TO DEVICE COMMUNICATION}

Communication between CCTV needs to be established to send SMS/MMS to the nearby CCTV or police station. So we want a CCTV to control another device. A device (CCTV 1) acts as a switch that reconfigures a second device (CCTV 2) to be "awake". One way for triggering the setup change is to do the following:

- Telemetry message is sent from Device 1 to Cloud Pub/Sub using Cloud IoT Core bridge

- Configure Cloud Function to get a message and send setup change

- $\quad$ Get setup change on Device 2

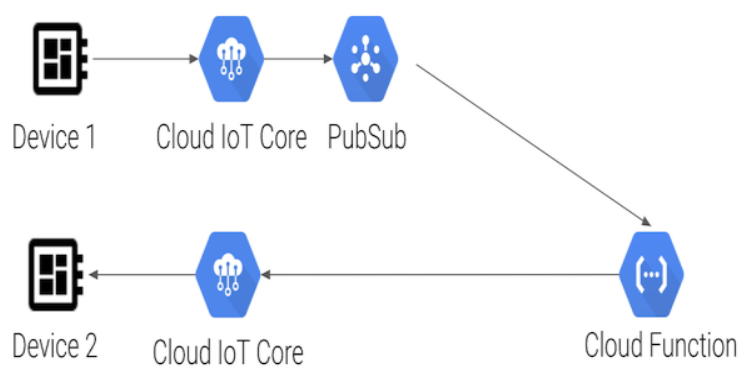

DEVICE TO DEVICE COMMUNICATION
A virtual device is employed for this method. When the virtual gadget interfaces, it transmits a telemetry message that is turned into a Cloud Pub/Sub message that reaches the Cloud Function. The Cloud Function then generates an asking message supported the telemetry information, that contains the written account and device IDs, set to the connecting device ID.

Because the connecting device ID is employed, the device is being returned a configuration amendment message from the Cloud IoT Core service. Once the device gets the message, it'll at that point send a second telemetry/measuring message to the server. This may then produce another setup alteration message that is transmitted back to the gadget.

\section{CONCLUSION}

Perceiving fights and vigorous behavior in the video is ansignificant area of implementation. Such capacity is likewise extremely supportive in video perception situations like in prison, mental or elderly facilities.Furthermore, reconnaissance by utilizing CCTV frameworks has achieved its best dimension. Likewise, sending data or information through information networks to servers is regular nowadays, however, coupling these two surveillance and information transmission process is an extremely testing activity. CCTV surveillance frameworks are for the most part realized and supervised by governments.In this way, utilizing CCTV system data do have a few disadvantages and are hard to deal with.In this paper, we proposed algorithms that can alarm the human administrator when an deserted suitcase, the presence of a hazardous actor anomalous conduct of an individualis detected. We have concentrate on restricting the number of fake alert.

\section{REFERENCES}

1. Febin I P, Jayasree K ,“A Survey of Computer Vision Based Methods for Violent Action Representation and Recognition".

2. Mike Haldas "'Send Security Camera Photos From Raspberry Pi Via Mms Text Message".

3. Malavika Nair, Mathew Gillroy, Neethu Jose, Jasmy Davies, "I-Surveillance Crime Monitoring and Prevention Using Neural Networks".

4. Peipei Zhou,Qinghai Ding, Haibo Luo,Xinglin Hou,"Violence Detection in Surveillance Video Using Low-Level Features".

5. E. Bermejo , O. Deniz , G. Bueno and R. Sukthankar,"Violence Detection in Video Using Computer Vision Techniques".

6. Q. Dai, J. Tu, Z. Shi, Y.-G. Jiang, and X. Xue., "Violent Scenes Detection Using Motion Features and Part-Level Attributes".

7. Daniel Moreira, Sandra Avila, Mauricio Perez, Daniel Moraes, ' Temporal Robust Features for Violence Detection".

8. A. Datta, M. Shah, and N. da Vitoria Lobo, "Person-on-person violence detection in video data.".

9. F. D. M. de Souza, G. C. Chavez, E. do Valle, D. A. Araujo, "Violence detection in video using spatio-temporal features".

10. M. Suresh Anand, M. Balamurugan,"Sugar Level Detection Using Thermal Images",International Journal of Engineering \& Technology, Volume 7, Issue no. 4.39, Dec 2018

11. T. Hassner, Y. Itcher, and O. Kliper-Gross, "Violent flows: Real-time detection of violent crowd behaviour."

12. R. Mehran, A. Oyama, and M. Shah, "Abnormal crowd behaviour detection using social force model. ".

13. H. Mousavi, S. Mohammadi, A. Perina, R. Chellali, and V. Murino, "Analysing tracklets for the detection of abnormal crowd behaviour".

14. K. Vijayakumar and C. Arun, "Continuous Security Assessment of Applications in Cloud Environment", International Journal of Control Theory and Applications, ISSN: 0974-5645 volume No. 9(36), Sep 2016, Page No. 533-541. 
15. R.Joseph Manoj, M.D.Anto Praveena, K.Vijayakumar, "An ACO-ANN based feature selection algorithm for big data", Cluster Computing The Journal of Networks, Software Tools and Applications, ISSN: 1386-7857 (Print), 1573-7543 (Online) DOI: 10.1007/s10586-018-2550-z, 2018.

16. K. Vijayakumar and V. Govindaraj, "An Efficient Communication Technique for Extrication and Cloning of packets on cloud", International Journal of Applied Engineering Research, ISSN 0973-4562 Vol. 10 No.66 May 2015

\section{AUTHORS PROFILE}

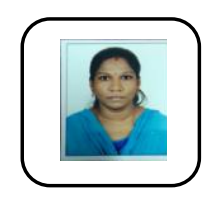

A.SANGEERANI DEVI B.Tech(IT),M.E(CSE) Assistant Professor, Department of Computer Science and Engineering, Sri Sairam Engineering College having $12+$ years of teaching experience. My area of interest is Data Mining and Big Data Analytics. Life Time member of ISTE, CSI, published 3 papers in Indian Journal of Public Health Research \& Development a scopus indexed journal.

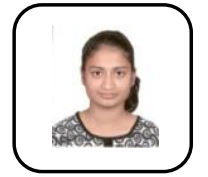

SONIA PRAKASH , BE(CSE), Student, Department of Computer Science and Engineering, Sri Sairam Engineering College. CSI Member. My Area of interest IOT, Blockchain and Computer Vision. Presented Paper in National Conference

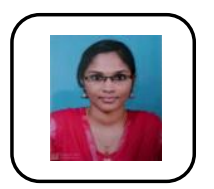

K.LAAVANYA, BE(CSE), Student, Department of Computer Science and Engineering, Sri Sairam Engineering College. CSI Member. My Area of interest IOT, Machine Learning and Computer Vision.

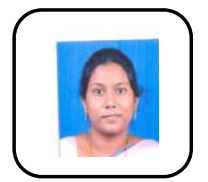

A.SHALI B.E.,M.E(CSE), Assistant Professor Department of Computer Science and Engineering, Sri Sairam Engineering College having 10+ years of teaching experience. My area of interest is Big Data Analytics and Cloud Computing.

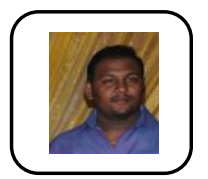

D.Satish Kumar, completed Bachelor degree (B.Tech) in Information Technology from anna University and Masters in Information Technology from Sathyabama University. Working as a Assistant Professor in Sri Sairam Engineering College, Chennai. Area of Research is networking and wireless communction. Life member of CSI, ISTE and IAENG. 